\title{
A PECULIAR FLARING EPISODE OF CYGNUS X-1
}

\author{
Wei Cui, Yu-Xin Feng, and Mark Ertmer \\ Department of Physics, Purdue University, West Lafayette, IN 47907 \\ Received 2001 November 19; accepted 2001 December 6; published 2001 December 24
}

\begin{abstract}
Recent monitoring of Cyg X-1 with the Rossi X-Ray Timing Explorer (RXTE) revealed a period of intense flaring, which started in 2000 October and lasted until 2001 March. The source exhibited some quite unusual behaviors during this period. The soft X-ray flux of the source went up and down 3 times on a timescale of about 1 month, as discovered by the All-Sky Monitor aboard RXTE, before finally returning to the normal level (of the hard state). The observed spectral and temporal X-ray properties of Cyg X-1 are mostly intermediate between the canonical hard and soft states. This is known previously for strong X-ray flares; however, we show that the source did enter a period that resembles, in many ways, a sustained soft state during the last of the three flares. We make detailed comparisons between this flare and the 1996 state transition in terms of the observed $\mathrm{X}$-ray properties, such as the flux-hardness correlation, X-ray spectrum, and power density spectrum. We point out the similarities and differences, and discuss possible implications of the results on our understanding of the phenomena of flares and state transitions associated with Cyg X-1.
\end{abstract}

Subject headings: binaries: general — stars: individual (Cygnus X-1) — X-rays: stars

\section{INTRODUCTION}

Cygnus X-1 has always been considered an archetypical black hole candidate (BHC; see reviews by Oda 1977, Liang \& Nolan 1984, and Tanaka \& Lewin 1995). Its observed spectral and temporal X-ray properties have, therefore, often been used to distinguish BHCs from their neutron star counterparts. Though flawed, this approach has resulted in the discovery of many BHCs whose candidacy is subsequently confirmed by dynamical measurements of the mass of the compact object.

Cyg X-1 shows two distinct spectral states: the hard state and the soft state. The source spends most of the time in the hard state where the soft X-ray $(2-10 \mathrm{keV})$ luminosity is relatively low and where the power-law X-ray spectrum is relatively flat. Once every few years, Cyg X-1 undergoes a transition from the hard state to the soft state. It remains in the soft state for weeks to months before returning to the hard state. During such a spectral state transition, the power-law spectrum "pivots" around 10-20 keV, causing a significant increase in the soft X-ray flux but a decrease in the hard X-ray $(\gtrsim 20 \mathrm{keV})$ (e.g., Zhang et al. 1997). The source has only occasionally been observed in the soft state (Oda 1977; Liang \& Nolan 1984; Cui et al. 1997a) and is, thus, not as well studied as in the hard state. Besides the difference in the spectral properties of the source between the two states, the temporal properties are also quite different. The power density spectrum (PDS) of Cyg X-1 in the hard state can be characterized by a white-noise component at low frequencies and a power-law component at high frequencies, with the characteristic "break frequency" in the range of $\sim 0.04-0.4 \mathrm{~Hz}$ (see reviews by van der Klis 1995 and Cui 1999). In the soft state, however, the PDS is dominated by " $1 / f$ " noise (Cui et al. 1997a). For both states, the PDS shows additional features at a few hertz (e.g., Belloni \& Hasinger 1990; Cui et al. 1997a). Moreover, the Xray flux of Cyg X-1 is known to vary on all timescales down to at least milliseconds; the X-ray variability seems stronger in the hard state than in the soft state.

Cyg X-1 is also known to experience frequent X-ray flares. While most flares last for less than a day, some can last for weeks to months, during which time additional short-duration flares may also occur. Studies have shown that at least some of the major flares are similar to state transitions (Pottschmidt et al. 2001; Y.-X. Feng, M. Ertmer, \& W. Cui 2001, in preparation). Therefore, the distinction between major flares and state transitions can sometimes be quite ambiguous. The X-ray flares of Cyg X-1 are poorly understood because of the lack of high-quality data. In this Letter, we present results from recent Rossi X-Ray Timing Explorer (RXTE) observations of Cyg X-1 during a peculiar period of intense flaring activities.

\section{ALL-SKY MONITOR OBSERVATIONS}

Starting in 2000 October, a series of long, intense X-ray flares was observed in Cyg X-1 by the All-Sky Monitor (ASM) aboard RXTE. Figure 1 (top panel) shows a portion of the ASM light curve of the source for this time period. Three major flares are clearly seen in the light curve. They all reached roughly the same peak flux and lasted for roughly the same length of time (about a month), although their time profiles are quite different. Figure 1 (middle panel) also shows a time series of the ratio of the count rate in the $3-5 \mathrm{keV}$ band to that in the $1.5-3 \mathrm{keV}$ band. Such a hardness ratio provides a rough measure of the X-ray spectral shape of the source. The results show that the spectrum of Cyg X-1 softens significantly during each of the flares. The minimum value of the hardness ratio is very similar for the first and last flares but seems higher for the middle flare (although the coverage of the later part of the middle flare is relatively sparse). It is interesting to note that both the peak flux and the minimum hardness ratio reached by the flares are nearly identical to those of the 1996 state transition of Cyg X-1 (Cui et al. 1997a). The question now is whether there are any clear distinctions between a flare that we see here and a canonical state transition of Cyg X-1.

To shed more light on the issue, we examined the correlation between the soft X-ray flux of the source and its spectral hardness, following the work of Wen, Cui, \& Bradt (2001). Those authors demonstrated, based mostly on the ASM data, that the flux-hardness correlation is a reliable indicator of the soft state, certainly more reliable than the soft X-ray flux alone. They found that the flux and hardness ratio are strongly correlated for the soft state but are weakly anticorrelated for the hard state (also see Li, Feng, \& Chen 1999). The transition between 


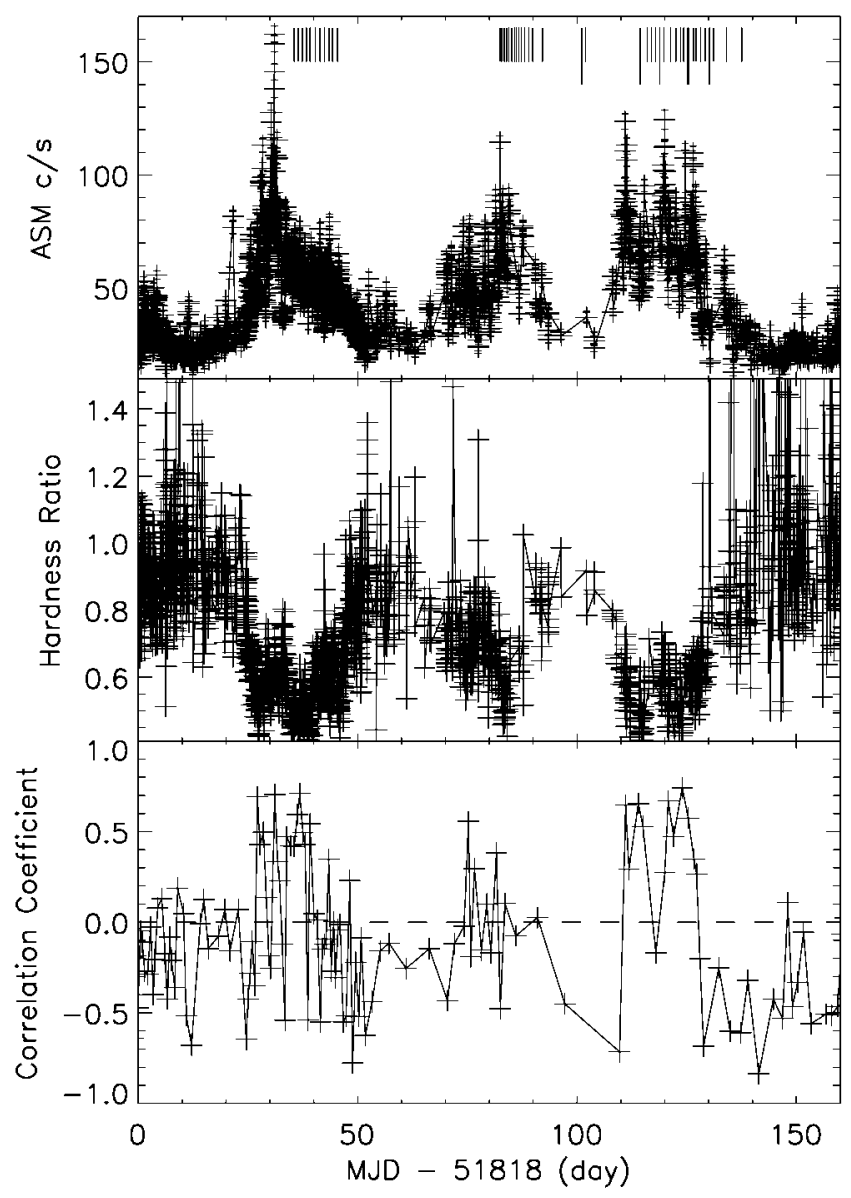

FIG. 1.-Top: ASM light curve (1.5-12 keV) of Cyg X-1. It comprises measurements from individual "dwells" with $90 \mathrm{~s}$ exposure times. The vertical lines at the top mark the times of the pointed observations, with the longer ones indicating those that were selected for Table 1. Middle: Time series of the ASM hardness ratio (3-12 keV/1.3-3 keV). Bottom: Correlation coefficient between the flux and the hardness ratio (see text).

the two states is a gradual and continuous process, as quantified by the correlation coefficient. We adopted the Spearman rankorder method (Press et al. 1992, p. 639) to investigate the correlation between the soft flux and the spectral hardness of the source, again following Wen et al. (2001). Briefly, both the rate and the hardness-ratio time series (with $90 \mathrm{~s}$ time bins, as shown in Fig. 1) were first parameterized with their rank numbers. The light curves were then broken into intervals that contain the same number of data points (18 in our case). Note that the intervals are not necessarily of the same duration because of the presence of coverage gaps. For each interval, a correlation coefficient between the two parameterized light curves was computed. The bottom panel of Figure 1 shows the evolution of the coefficient throughout the flaring period.

The results show a weak, negative correlation during nonflare intervals, indicating that the source reached the hard state (Wen et al. 2001). The most striking feature, however, is an interval of positive correlation (except for a dip in the middle) during the last flare. Interestingly, the average value of the coefficient for this flare is about the same as that for the 1996 state transition (see Wen et al. 2001). Therefore, the last flare seems quite similar to the 1996 soft state. However, the other two flares behave quite differently. Although roughly the same positive coefficient is achieved at times during the first flare, the fluctuation is quite large. The middle flare seems to be intermediate between the hard and soft states. We speculate that flares and state transitions may differ only in a quantitative sense; the underlying physical process(es) may be common for these seemingly different phenomena. To make progress on these issues, we obtained detailed X-ray properties of the source during the last flare, using data from pointed observations, and compared the properties with those during the 1996 state transition.

\section{POINTED RXTE OBSERVATIONS}

During the flaring period, Cyg X-1 was frequently observed by the large area detectors aboard RXTE, namely, the Proportional Counter Array (PCA), which covers a nominal energy range of 2-60 keV, and the High Energy X-ray Timing Experiment (HEXTE), which covers a nominal energy range of 15$250 \mathrm{keV}$. The times of the pointed observation are indicated by tick marks in Figure 1. These observations provided data for the detailed examination of the spectral and timing properties of the source during the last flare; these properties were then compared with those observed during the 1996 state transition.

\subsection{Spectral Analysis and Results}

For each observation, we constructed an X-ray spectrum of Cyg X-1 from the PCA and HEXTE data with FTOOLS (v5.0) as well as the calibration files and background models that accompanied this release of the software. To minimize calibration uncertainties associated with the PCA, we only used data from the first xenon layer of each detection unit (which is most accurately calibrated). The trade-off is that we lose spectral coverage at energies above roughly $30 \mathrm{keV}$. On the other hand, we could rely on the HEXTE data to extend the coverage to higher energies. At low energies $(\leq 5 \mathrm{keV})$, the calibration of the PCA is also relatively uncertain. To be conservative, therefore, we ignored data below about $4.5 \mathrm{keV}$ for spectral analyses.

For technical reasons, not all five detector units of the PCA were turned on for the observations. The number of units in use varied from one observation to the other, so did the specific set of detector units. For a given observation, we used Standard 2 data to produce a spectrum for each detector unit. We then derived a background spectrum for that unit and subtracted it from the observed spectrum to obtain a source spectrum for spectral modeling. Similarly, we constructed an overall spectrum, along with a background spectrum, for each of the two HEXTE clusters. The individual background-subtracted spectra were then modeled jointly in XSPEC (v11.0). We allowed the relative normalizations of the detector units to vary in the fits, to account for any slight difference among the units of each instrument and the known difference between the two instruments. We also added $1 \%$ systematic uncertainty to the data.

To facilitate a quantitative comparison between the last flare observed here and the 1996 state transition, we simply adopted the empirical model used by Cui et al. (1997a) to fit the spectra. However, we did not include a disk blackbody component because, by ignoring data below $\sim 4.5 \mathrm{keV}$, we were no longer able to reliably constrain it. The model now consists of a broken power law with a high-energy rollover. In order to achieve acceptable fits, however, we also had to include a Gaussian function in the model to account for residuals between 5 and $8 \mathrm{keV}$ for all observations. The centroid energy of the Gaussian component varies in the range of $6.3-6.6 \mathrm{keV}$, which seems to indicate the presence of an Fe $\mathrm{K}$ emission line. For the fits we 
TABLE 1

Key Spectral Parameters for Selected Observations

\begin{tabular}{|c|c|c|c|c|c|c|c|c|}
\hline \multirow[b]{2}{*}{ Number } & \multirow[b]{2}{*}{ OBSERVATION ID ${ }^{a}$} & \multicolumn{3}{|c|}{ Broken Power Law ${ }^{b}$} & \multicolumn{2}{|c|}{ High-ENERgy CUTOFF $^{c}$} & \multirow[b]{2}{*}{$\chi_{\nu}^{2} / \mathrm{dof}$} & \multirow[b]{2}{*}{ FLuX $^{\mathrm{d}}$} \\
\hline & & $\alpha_{1}$ & $\alpha_{2}$ & $\begin{array}{c}E_{b} \\
(\mathrm{keV})\end{array}$ & $\begin{array}{c}E_{c} \\
(\mathrm{keV})\end{array}$ & $\begin{array}{c}E_{f} \\
(\mathrm{keV})\end{array}$ & & \\
\hline$\ldots \ldots$ & $03-13-00$ & $1.98_{-0.08}^{+0.03}$ & $1.60_{-0.02}^{+0.01}$ & $10.5_{-0.2}^{+0.4}$ & $22_{-2}^{+1}$ & $152_{-8}^{+7}$ & $1.05 / 232$ & 3.07 \\
\hline$\ldots \ldots$ & 01-05-01 & $2.51_{-0.04}^{+0.02}$ & $1.81_{-0.02}^{+0.01}$ & $10.7_{-0.1}^{+0.1}$ & $24_{-1}^{+1}$ & $130_{-7}^{+6}$ & $1.17 / 229$ & 2.05 \\
\hline$\ldots \ldots$ & 01-07-00 & $2.66_{-0.03}^{+0.02}$ & $2.00_{-0.01}^{+0.01}$ & $10.7_{-0.1}^{+0.1}$ & $21_{-1}^{+1}$ & $109_{-4}^{+6}$ & $1.32 / 278$ & 3.32 \\
\hline$\ldots \ldots$ & 01-08-05 & $2.74_{-0.03}^{+0.01}$ & $1.96_{-0.02}^{+0.01}$ & $10.5_{-0.1}^{+0.2}$ & $22_{-1}^{+1}$ & $123_{-6}^{+5}$ & $1.21 / 228$ & 2.74 \\
\hline$\ldots \ldots$ & 01-09-00 & $2.54_{-0.05}^{+0.01}$ & $1.90_{-0.02}^{+0.01}$ & $10.7_{-0.1}^{+0.2}$ & $21_{-2}^{+3}$ & $144_{-14}^{+12}$ & $1.16 / 228$ & 3.52 \\
\hline $6 \ldots \ldots$ & 01-09-05 & $1.86_{-0.06}^{+0.12}$ & $1.55_{-0.02}^{+0.02}$ & $10.6_{-0.4}^{+0.6}$ & $19_{-2}^{+2}$ & $125_{-8}^{+8}$ & $1.00 / 236$ & 2.45 \\
\hline
\end{tabular}

a The prefix "50109-" is omitted.

${ }^{\mathrm{b}}$ The soft and hard power-law photon indices are $\alpha_{1}$ and $\alpha_{2}$, respectively, and $E_{b}$ is the break energy.

${ }^{\mathrm{c}} E_{c}$ and $E_{f}$ are the cutoff energy and the $e$-folding energy, respectively.

${ }^{\mathrm{d}}$ The observed 5-200 keV flux (in units of $10^{-8} \mathrm{ergs}^{-2} \mathrm{~s}^{-1}$ ).

also fixed the hydrogen column density at $6.2 \times 10^{21} \mathrm{~cm}^{-2}$ (Schulz et al. 2002; Bałucińska \& Hasinger 1991), again becasue of the lack of sensitivity of the RXTE data at low energies. The best-fit parameters are listed in Table 1 for a selected sample of observations that cover the last flare (see Fig. 1 for the times of these observations). The uncertainties shown represent $90 \%$ confidence intervals. Figure 2 illustrates the quality of a representative fit.

The results show the clear spectral evolution of the source over the last flare. The spectrum softens progressively as the source flux increases toward the peak of the flare and hardens as the flux decreases from the peak; the two transitional periods seem to mirror each other (although the coverage of the "lowto-high" transition is poorer). At the peak, the parameters of the third and fourth observations are nearly identical to those of the 1996 soft state (see the results of the fourth observation in Table 2 of Cui et al. 1997a). In fact, the spectrum of the source is similarly soft during all observations between (and around) these two selected ones (see Fig. 1 for the times of other observations). Therefore, this flare is spectrally very similar to the 1996 state transition. However, one difference is noticeable: during the 1996 episode, the flux-hardness correlation stayed positive throughout the soft state (although the coverage was not very good; Cui et al. 1997b; Wen et al. 2001). During the flare, even when the flux-hardness correlation is positive (see Fig. 1), the spectrum of the source is at times

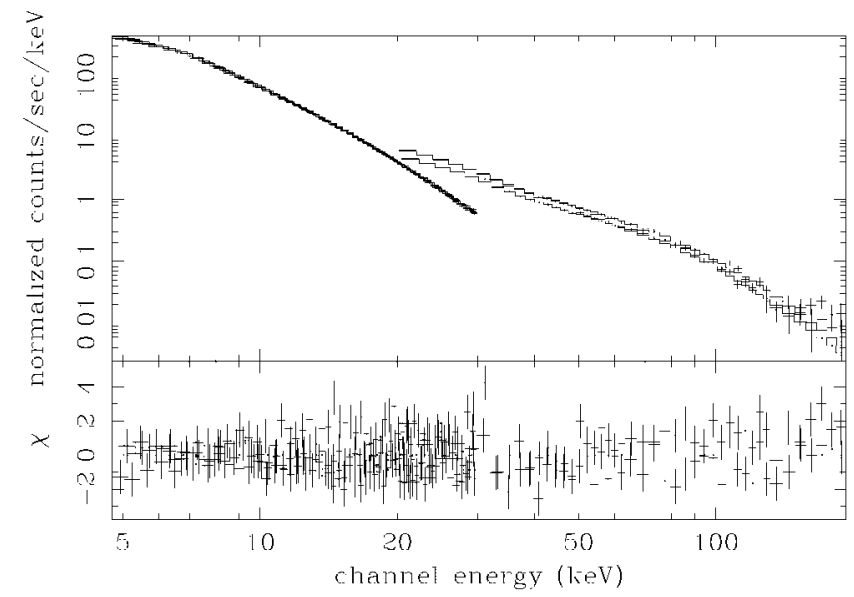

FIG. 2.-Combined PCA/HEXTE spectrum of Cyg X-1 for the third observation in Table 1. The best-fit model is shown in the solid histogram. The bottom panel shows the residuals of the fit. significantly harder (e.g., comparing the second and fifth observations with the third and fourth observations in Table 1).

\subsection{Timing Analysis and Results}

We also proceeded to examine the temporal properties of Cyg X-1 by using data from high timing resolution modes (single-bit and event). For each observation, we rebinned the event data to $2^{-13} \mathrm{~s}$ time bins, which is the resolution of the single-bit data, and then combined all the data to cover the entire PCA passing band. We broke the light curve into $128 \mathrm{~s}$ segments and carried out a $2^{20}$ point fast Fourier transformation of each segment to obtain the corresponding PDS that is Leahy-normalized (Leahy et al. 1983). The power spectra of all segments were then weighted and co-added to obtain the average PDS, as well as its variances. From the average PDS we subtracted the dead-time-corrected power due to Poisson statistical fluctuation (see Zhang et al. 1995 for discussion on dead-time effects). Finally, we divided the resulting PDS by the mean source rate to express Fourier powers in terms of fractional rms amplitudes (van der Klis 1995). The results are presented in Figure 3 for the same selected observations (as in Table 1).

Over the duration of the last flare (see Fig. 1), the PDS of the source evolved significantly. The shape of the PDS went from being "flat-topped" at the beginning, which is characteristic of the hard state, to being roughly $1 / f$ at the peak, which is characteristic of the soft state; it returns to the flat-topped profile at the end, completing a full transition. In between, however, the PDS shows a mixture of the flat-topped component and the $1 / f$ component, which is characteristic of the transitional periods between the hard and soft states (Cui et al. 1997a, 1997b). There are clearly additional features. For the third and fourth observations, the PDS further steepens at roughly $10 \mathrm{~Hz}$, which was observed in the 1996 soft state (Cui et al. 1997a). For other observations, there is a broad but localized feature centered at a few hertz, again similar to what has been observed during the hard state (e.g., Belloni \& Hasinger 1990) and transitional periods in 1996 (Cui et al. 1997a). Figure 3 also shows the overall variability of the source at different stages of the flare. The fractional rms amplitude shows a decreasing trend toward the peak, although the change is quite small compared with that for transient BHCs during a state transition (van der Klis 1995). We conclude that the flare is also similar to the 1996 state transition in terms of the observed temporal properties of Cyg X-1. 


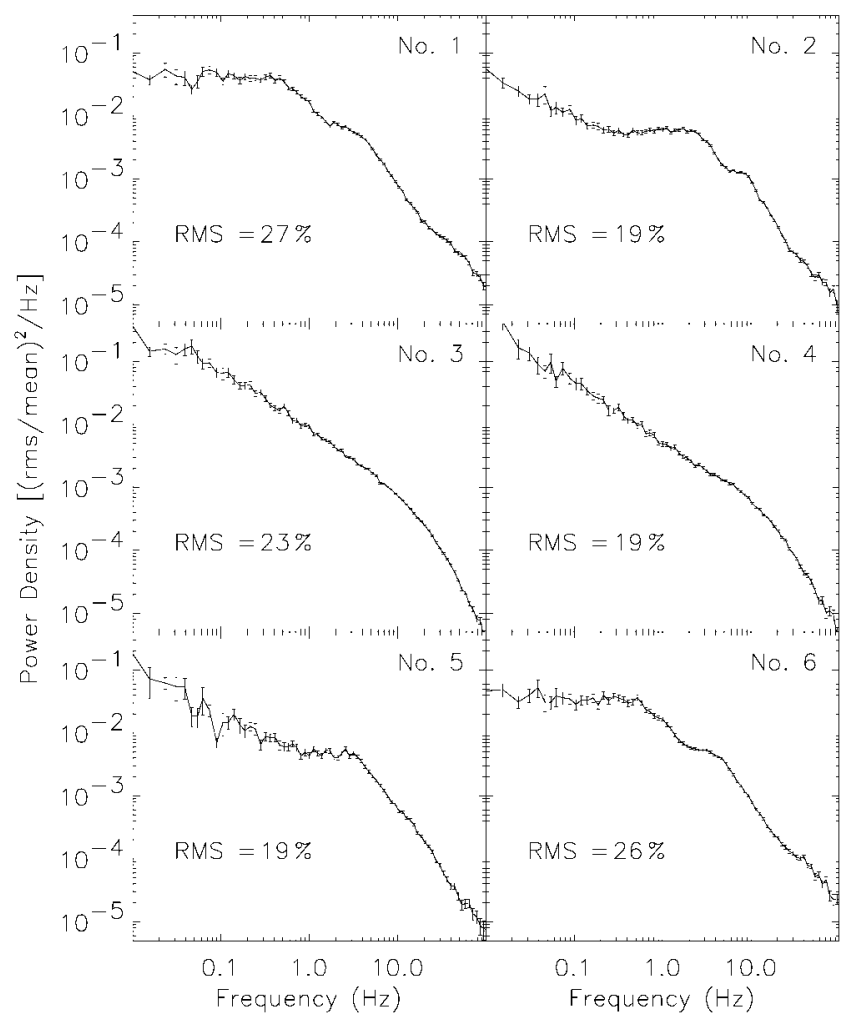

FIG. 3.-PDSs of Cyg X-1 for a select sample of observations (see Table 1). The overall fractional $\mathrm{rms}$ amplitude $(0.02-32 \mathrm{~Hz})$ is also shown for each observation.

\section{SUMMARY AND DISCUSSION}

We observed Cyg X-1 during an unusual period when three consecutive major X-ray flares occurred over a period of about 5 months. Following each flare, the source did seem to completely return to the hard state before the next one started, as indicated by the flux-hardness correlation. The correlation also indicates that $\mathrm{Cyg} \mathrm{X}-1$ entered a sustained period during the last flare that is very similar to the 1996 soft state. This is supported by striking similarities between the observed spectral and temporal properties of the source during that time period and those during the 1996 soft state (although there are some differences). Therefore, we argue that the physical process that is responsible for triggering a flare or a state transition is likely to be the same. The difference between the two types of phenomena seems to be mostly quantitative. For instance, the soft state lasted for only about a week during the flare, compared with more than 2 months in the 1996 episode. This difference might be due to the difference in the change of physical quantities, such as the fraction of accretion energy dissipated in the disk.

Although only an empirical model was used for spectral studies, the results do clearly reveal the evolution of the source, thanks to the improved coverages of the transitions. It was known previously that a broken power law would be required to model the spectrum of Cyg X-1 at high energies (Ebisawa et al. 1996; Cui et al. 1997a), as opposed to a simple power law for most BHCs (Tanaka \& Lewin 1995). Not only did we confirm this in our investigation, we also quantified the evolution of this component throughout the transitions. Moreover, we confirmed the presence of a "settling period" after the lowto-high transition or before the high-to-low transition, as suggested by Cui et al. (1997a), when the soft flux is at the softstate level but the spectral and timing properties are still intermediate between the hard and soft states. Such a period manifests itself prominently in the PDS shape. Finally, throughout the flare the observed 5-200 keV flux does not vary significantly. This is again consistent with previous results from a study of the 1996 state transition (Zhang et al. 1997).

We conclude that perhaps we can learn a great deal about the origin of rare state transitions of Cyg X-1 from studying more frequently occurring X-ray flares. We have tried to establish a connection between the two types of phenomena in this investigation. The next step will be to carry out, in a more systematic manner, detailed comparisons between a range of X-ray flares and state transitions (Y.-X. Feng, M. Ertmer, \& W. Cui 2001, in preparation).

This work was supported in part by NASA through grants NAG 5-9098 and NAG 5-9998.

\section{REFERENCES}

Bałucińska, M., \& Hasinger, G. 1991, A\&A, 241, 439

Belloni, T., \& Hasinger, G. 1990, A\&A, 227, L33

Cui, W. 1999, in ASP Conf. Ser. 161, High-Energy Processes in Accreting Black Holes, ed. J. Poutanen \& R. Svensson (San Francisco: ASP), 97

Cui, W., Heindl, W. A., Rothschild, R. E., Zhang, S. N., Jahoda, K., \& Focke, W. 1997a, ApJ, 474, L57

Cui, W., Zhang, S. N., Focke, W., \& Swank, J. 1997b, ApJ, 484, 383

Ebisawa, K., Ueda, Y., Inoue, H., Tanaka, Y., \& White, N. E. 1996, ApJ, 467, 419

Leahy, D. A., Darbro, W., Elsner, R. F., Weisskopf, W. C., Sutherland, P. G., Kahn, S., \& Grindlay, J. E. 1983, ApJ, 266, 160

Li, T. P., Feng, Y.-X., \& Chen, L. 1999, ApJ, 521, 789

Liang, E. P., \& Nolan, P. L. 1984, Space Sci. Rev., 38, 353

Oda, M. 1977, Space Sci. Rev., 20, 757

Pottschmidt, K., Wilms, J., Nowak, M. A., Heindl, W. A., Smith, D. M., \& Staubert, R. 2000, A\&A, 357, L17
Press, W. H., Teukolsky, S. A., Vetterling, W. T., \& Flannery, B. P. 1992, Numerical Recipes in C (2d ed.; Cambridge: Cambridge Univ. Press)

Schulz, N. S., Cui, W., Canizares, C. R., Marshall, H. L., Lee, J. C., Miller, J. M., \& Lewin, W. H. G. 2002, ApJ, 565, 1141

Tanaka, Y., \& Lewin, W. H. G. 1995, in X-Ray Binaries, ed. W. H. G. Lewin, J. van Paradijs, \& E. P. J. van den Heuvel (Cambridge: Cambridge Univ. Press), 126

van der Klis, M. 1995, in X-Ray Binaries, ed. W. H. G. Lewin, J. van Paradijs, \& E. P. J. van den Heuvel (Cambridge: Cambridge Univ. Press), 252

Wen, L., Cui, W., \& Bradt, H. V. 2001, ApJ, 546, L105

Zhang, S. N., Cui, W., Harmon, B. A., Paciesas, W. S., Remillard, R. E., \& van Paradijs, J. 1997, ApJ, 477, L95

Zhang. W., Jahoda, J., Swank, J. H., Morgan, E. H., \& Giles, A. B. 1995, ApJ, 449, 930 